\title{
L'éthique et l'art du soin
}

\section{Lazare Benaroyo}

Prof. Dr med. et Dr phil., membre de la rédaction, Faculté de biologie et de médecine, et Plateforme interdisciplinaire d'éthique, Université de Lausanne

Le corps humain, écrit Maurice Merleau-Ponty, est un entrelacs de nature et d'existence [1]. Dans l'état de bonne santé, il est vécu comme allant de soi, comme une évidence. Lorsque qu'il se fissure, qu'il se désintègre, l'existence ne va plus de soi, l'état de mal-être est ressenti, et la quête médicale commence.

Comment le médecin va-t-il répondre à l'appel à l'aide du malade? En miroir de cet entrelacs, il va d'une part se fonder sur une approche scientifique, cherchant à expliquer la cause du dysfonctionnement du corps, "chose parmi les choses», et à maîtriser dans le même mouvement le cours de la maladie; d'autre part, il va adopter une attitude personnelle, cherchant à comprendre la signification de la brisure de l'être-aumonde qui vient rompre l'harmonie de l'existence jusqu'alors insouciante de la personne malade [2]. D'un point de vue clinique, cette double réponse relève en premier lieu de l'art, au sens d'un savoir pratique, d'une sagesse pratique, c'est-à-dire de l'aptitude à prendre la décision la mieux adaptée, la meilleure possible, dans une situation singulière de soin. D'un point de vue académique, la médecine est conçue comme une science qui permet de fonder la pratique et de lui donner les moyens d'être efficace au plan thérapeutique, préventif et pronostic. Art ou science? La pratique médicale cherche à conjuguer ces deux pôles, parfois en tension, souvent au cœur de malentendus. Quel regard l'éthique et la philosophie peuvent-elles porter sur cette question?

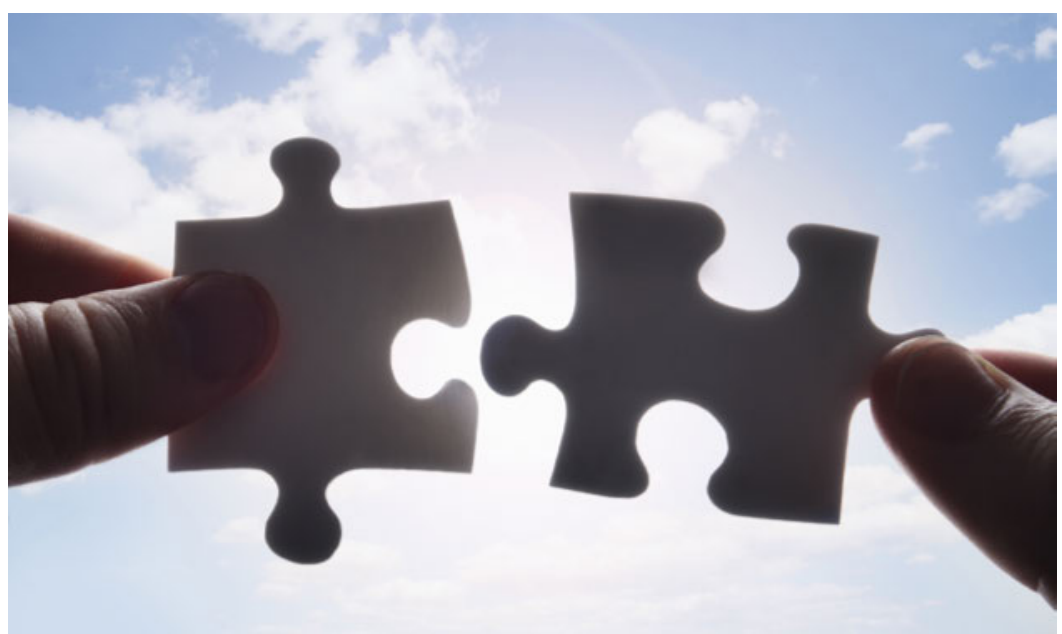

L'art de l'écoute est un défi auquel tout soignant est confronté: il suppose une ouverture à l'autre.
En explorant ces relations, cet essai cherche à mieux comprendre quelle place et quel sens l'art médical occupe aujourd'hui et quelle contribution l'éthique peut apporter à une meilleure compréhension de sa pertinence.

\section{Perspective historique}

D'un point de vue historique, cette question est récente: si l'art et la science médicales étaient alignés autour de la même visée éthique de soin dans l'Antiquité grecque jusqu'aux temps modernes, l'importance prise par la démarche scientifique dès le XVII ${ }^{\mathrm{e}}$ siècle et surtout dès le début du XX $\mathrm{XX}^{\mathrm{e}}$ siècle, a troublé cette articulation homogène au détriment d'une dissociation entre art et science.

\section{Art ou science? La pratique médicale cherche à conjuguer ces deux pôles, parfois en tension, souvent au cœur de malentendus.}

Dans la perspective inaugurée par la science moderne, la médecine s'attache principalement dès le milieu du $\mathrm{XIX}^{\mathrm{e}}$ siècle à définir les événements pathologiques comme des données qu'elle peut contrôler pour en modifier la structure et en maîtriser l'évolution, notamment les perturbations biologiques dont la démonstration a pu prouver expérimentalement qu'elles sont la cause des maladies. Le rétablissement de la santé fait alors appel à une éthique de la compétence technique tournée vers la restauration des troubles biologiques considérés comme étant la source des perturbations de la santé. L'art médical classique, en tant que savoir pratique orienté vers la globalité, trouvait moins sa place dès la fin du XIX siècle au sein d'un ethos médical qui tirait sa force de la fragmentation du savoir et de la différentiation disciplinaire permettant la spécialisation dans l'univers de la pathologie d'organes. L'attention était désormais portée sur l'organe, le tissu, la cellule, aujourd'hui la molécule, considérés comme les espaces au sein desquels se développe la source du mal à combattre, ceci au dépens d'une attention portée à la manière dont ces perturbations troublent l'équilibre de l'organisme dans son ensemble [3].

Sous cet angle, l'art médical a pris la figure d'une évaluation clinique imprécise à l'aune de la subjectivité du soignant, au point d'être jugée péjorativement au 
tournant du $\mathrm{XX}^{\mathrm{e}}$ siècle. L'éminent professeur de médecine à l'Université d'Harvard Richard Cabot dira en 1926 qu'il est temps que les sciences médicales et infirmières s'orientent dorénavant vers une conception du soin compris comme une curing attitude, une aptitude à guérir, en renonçant à la vision du soin reposant sur une prise en charge globale dans la mesure où «l'attention qui doit être portée au diagnostic demande déjà suffisamment de concentration pour porter également une attention à tout ce qui l'entoure» [4]. La médecine doit se concentrer sur le corps, disait-il, les autres dimensions relèvent d'autres disciplines.

Cette affirmation a suscité au cours des années 1920, puis de manière plus marquée au cours des années 1980, une forte réaction de la part de médecins qui déploraient cette nouvelle conception du soin et l'effacement de l'art médical au profit de l'exclusive compétence technique. Ce mouvement plaidait vigoureusement pour une «humanisation» de la médecine au nom d'une crise de l'éthique médicale [5]. Nous sommes face à un paradoxe, disaient ces derniers: le soin doit précéder l'acte technique, alors que c'est l'acte technique qui guide maintenant le soin.

L'attention portée aux pathologies d'organes fait appel à l'aptitude à classer et à catégoriser, alors que l'art du soin, portant sur l'organisme dans son ensemble, fait appel à la capacité du soignant de juger et d'évaluer moralement la situation. Dans la mesure où ces deux conceptions du vivant sont indispensables et indissociables dans le cadre d'un bon soin, il est impératif, ajoutaient-ils, de redonner à nouveau vie à une conception du soin qui porte la médecine à la hauteur de sa mission et qui conjugue science et art, au sens de l'aptitude à prendre en compte l'organisme dans sa globalité, au-delà de la lésion d'organe. Une conception du soin qui intègre en somme les deux registres de compréhension du vivant: le registre du vivant en tant qu'organe et du vivant en tant qu'organisme. Il s'agissait de faire revivre autour des années 1980 les tentatives déjà entreprises de réaliser cette articulation au cours des années 1920 à travers le développement d'une anthropologie clinique [6].

Francis Peabody, un collègue de Cabot à Harvard, écrivait en ce sens en 1927: "One of the essential qualities of the clinician is interest in humanity, for the secret of the care of the patient is in caring for the patient» [7]. De nombreux auteurs, tels William Osler par exemple, se joignaient à Peabody pour développer une clinique humaniste à même de porter une égale attention aux dimensions techniques et aux enjeux humains qui sont mobilisés dans l'état de maladie, en relevant l'importance de pouvoir rétablir l'équilibre de l'organisme, tout autant que de corriger le dysfonctionnement d'or- gane, afin de redonner à la personne malade la possibilité de vivre une existence où il ou elle peut déployer ses potentialités et retrouver sa liberté.

Au cours des années 1980, cette vision du soin réapparaît dans le cadre du projet de développement de l'éthique du soin (Ethics of Care) [8]. Ces efforts visaient à affronter le défi auquel tout soignant est confronté aujourd'hui: articuler les deux registres qui traversent le champ du soin. L'art de l'écoute suppose une mise en retrait, une ouverture à l'autre, une non-maîtrise alors que l'usage de la technique est fondé sur la mấtrise et le contrôle [9].

Ce paradoxe, qui habite le soin médical, est inhérent à l'entrelacement de l'humain et de la technique. La personne souffrante devrait pourtant être appréhendée, comprise et soignée au-delà de ce paradoxe. La sagesse pratique consiste dès lors à la fois à écouter et à entendre ce que le malade nous dit [10]. Comme le relèvent Schwartz et Wiggins, adopter de front ces deux attitudes ne va cependant pas de soi, dans la mesure où elles font appel à des formes de connaissances et à des méthodes différentes, qui entretiennent des relations paradoxales [5]. Confronté à l'universel et au particulier, à l'objectif et au subjectif, au désir d'infini en regard d'une réalité finie, le clinicien contemporain est ainsi confronté au défi de toujours situer son agir technique dans le projet de soin qui le porte, en acceptant que la pratique médi-

L’attention portée par le soignant à la préservation de cet inconnaissable ouvre au patient un horizon de reconnaissance et de confiance.

cale - tout en étant excellente au plan technique - a une limite; cette limite, c'est l'autre. La pensée du philosophe Emmanuel Levinas semble particulièrement féconde pour affronter ce défi, dans la mesure où elle accorde une attention particulière à l'asymétrie qui caractérise une relation de soin au plan éthique.

\section{Vers quelle ressource éthique puiser?}

Il y a en effet une part d'inconnaissable chez le patient que la technique ne peut pas maîtriser et qu'il est important de préserver, pour laisser s'épanouir la liberté qui cherche à retrouver son chemin au cœur de l'existence troublée du malade. L'attention portée par le soignant à la préservation de cet inconnaissable ouvre au patient un horizon de reconnaissance et de confiance, qui conforte ce dernier dans l'idée que sa souffrance lui appartient. C'est à ce moment-là que les traces de son altérité peuvent apparaître et qu'il peut avoir le sentiment du profond respect qui lui est ainsi adressé. C'est justement sur ce point que la pensée d'Emmanuel 
Levinas peut nourrir la réflexion sur la responsabilité éthique du soignant [11].

Une lecture levinassienne de la relation clinique enjoint en effet à percevoir la responsabilité soignante comme une attention particulière portée à la "visitation" du patient, ne réduisant pas le colloque singulier - qui conduit du diagnostic au traitement et au pronostic-, à ses aspects techniques. En d'autres termes, cela signifie que l'éveil éthique du soignant consiste à accepter la profonde altérité de son patient, qui s'exprime à travers son visage et à travers la vulnérabilité qui y est associée. La vulnérabilité non pas en tant que perte d'autonomie à combler dans la mesure du possible par la médecine, mais bien plus en tant qu'appel, qu'injonction éthique à prendre soin de ce patient-là, en ouvrant un espace où l'avenir est possible pour lui. Cette conception de la responsabilité fait appel à une capacité d'accueil, à une hospitalité du soignant qui mobilise sa propre vulnérabilité. C'est au cœur de ce dialogue entre deux vulnérabilités que la confiance peut prendre naissance et se déployer dans une prise en soin singulière à même de répondre de manière responsable au noyau de la souffrance telle qu'elle est perçue par le malade [12].

Cependant, penser avec Levinas implique un décentrement chez le soignant, dans la mesure où ce dernier doit faire appel à d'autres approches que la seule dimension technique - sans pour autant nier ou amoindrir cette dernière, au contraire; mais ce n'est pas la technique qui pourra définir le projet de soin. Les sciences humaines, la dimension relationnelle, doivent intervenir en amont. Cela implique d'entrer en dialogue avec la personne sur un autre mode que le pur mode anamnestique traditionnel. Il importe dans ce contexte de déployer plusieurs formes d'anamnèse successives pour respecter les temporalités en présence. Il arrive en effet qu'un soignant s'inscrive dans une temporalité technique et rencontre un patient dans la même temporalité. Mais parfois, alors même que la temporalité technique est fortement présente du côté du soignant, engagé dans l'action de soin à mener, le patient, pour sa part, se situe dans une toute autre temporalité: «Mais que vais-je devenir?» Il ne s'agit pas alors de lui dire: tel examen va répondre à la question. Ce n'est pas la technique qui va répondre à la question, c'est le soignant attentif à la temporalité du patient qui pourra le faire. C'est à ce moment-là que les traces de l'altérité du malade commencent à apparaître et qu'il aura le sentiment que le ou les soignants s'intéresse(nt) à lui, pour lui-même. C'est souvent quand on ne parle pas - au moment de l'auscultation par exemple - que le patient nous fait part de sentiments importants à ses yeux. Lécouter nous permettrait d'accéder à ces traces. Or, que dit-on habituellement? «Excusez-moi, pourriez-vous faire le silence, car je dois ausculter...» [9]. C'est justement à ce moment-là qu'il faudrait pouvoir écouter très attentivement...

\section{Perspective clinique}

Le défi que doit dès lors relever une éthique du soin aujourd'hui nous semble être celui d'aborder de front les paradoxes inhérents à la pratique de la médecine dans le cadre d'une éthique de l'attention et de la réponse qui déploie les divers registres du soin au sein d'une sagesse pratique [10]. Cette démarche nous semble constituer une posture de veille indispensable qui engage la responsabilité éthique du soignant au cœur même de son activité. En ce sens, clinique et éthique constituent les deux figures d'un même agir spécifique. C'est en affrontant patiemment ce défi dans le cadre d'une philosophie du soin [13] que la clinique nous semble pouvoir se déployer à sa juste mesure.

\section{Crédit photo}

(c) Mikdam | Dreamstime.com

\section{Références}

1 Merleau-Ponty M. Le visible et l'invisible, Paris: Gallimard, 1964, p. 178-82.

2 Zaner RM. Ethics and the Clinical Encounter, Englewood Cliffs, N.J., Prentice Hall, 1988.

3 Jewson ND. The disappearance of the sick-man from medical cosmology, 1170-1870, Sociology. 1976;10(2):225-44.

4 Cabot R. Adventures on the Borderlands of Ethics, New York: Harper and Brothers, 1926, p. 16.

5 Schwartz MA and Wiggins O. Science, humanism, and the nature of medical practice: a phenomenological view, Perspectives in Biology and Medicine. 1985;28(3):331-61.

6 Benaroyo L, Kurt Goldstein. La médecine en dialogue avec la vie: l'approche de l'anthropologie clinique des années 1930, in Lefève C, Mino JC, Zaccai N. Le soin, approches contemporaines, Paris: PUF, 2016, p. 49-60.

7 Peabody F. The care of the patient, Journal of the American Medical Association. 1927;88(12):877-82.

8 Reich W (ed). History of the notion of care, Encyclopedia of Bioethics, New York, McMillan, (5 vol.) 1995.

9 Baron RJ. An introduction to Medical Phenomenology: I Can't Hear You While I'm Listening, Annals of Internal Medicine 103, 1985, pp. 606-11.

10 Benaroyo L. Constructing medical wisdom. The significance of Healing narratives for clinical practice, in Neschke A, Sepp AH (ed.). Sprache und Wissenserwerb Language and Acquisition of Knowledge, Nordhausen: Traugott Bautz;2011. p. 233-44.

11 Benaroyo L. Le visage au-delà de l'apparence. Levinas et l'autre rive de l'éthique. Lo Sguardo. 2016;20(1):217-23.

12 Benaroyo L. Soin, confiance et disponibilité: les ressources éthiques de la philosophie d'Emmanuel Lévinas, Ethique et Santé 1 , 2004, pp. 60-3.

13 Benaroyo L, Lefève C, Mino JC, Worms F. La philosophie du soin. Ethique, médecine et société. Paris: PUF, 2010. 